\title{
Rayleigh Scattering of Mössbauer Radiation Method Used in Dynamics Studies of Condensed Matter
}

\author{
S.E. ENESCU ${ }^{a}$ AND I. BIBICU ${ }^{b, *}$ \\ a "Horia Hulubei" National Institute for Physics and Nuclear Engineering \\ IFIN-HH, P.O. Box MG-6, 76900 Bucharest-Magurele, Romania \\ ${ }^{b}$ National Institute for Material Physics \\ P.O. Box MG-7, 76900 Bucharest-Magurele, Romania
}

(Received November 2, 2004)

\begin{abstract}
The Rayleigh scattering of the Mössbauer radiation is a less common technique used in the study of the properties of condensed matter. The detection of the scattered radiation via the nuclear resonance absorption, with an energy resolution of the order of $10^{-8} \mathrm{eV}$ makes this technique very sensitive to dynamics changes. The results of our studies by the Rayleigh scattering of the Mössbauer radiation on pyrolytic graphite $(\mathrm{C})$ and rubidium tetrachlorozincate $\left(\mathrm{Rb}_{2} \mathrm{ZnCl}_{4}\right)$ are reported. The contribution of the coherent inelastic scattering to the total intensity in $\mathrm{C}(002)$ is presented like a consequence of the coupling motions of $\mathrm{C}$ atoms in the hexagonal plane. The normal-incommensurate phase transition in $\mathrm{Rb}_{2} \mathrm{ZnCl}_{4}$ is discussed in connection with the photon-phonon interaction.
\end{abstract}

PACS numbers: 33.45.+x, 64.60.-i, 64.70.Rh

\section{Introduction}

Scattering experiments have given us a lot of information about the microscopic behavior of an enormous variety of condensed matter systems. The excitation energies of interest in solid state physics range from tens of electronvolts down to tenth of millielectronvolts, zero-energy transfer being a special case. The angular dependence of the scattering cross-section in this zero-energy limit gives us the Fourier transform of the static spatial arrangement of the atoms making up the condensed matter system. This scattering is called the Bragg scattering in a perfect crystal. Because of the enormous variety of excitations, extending from

*corresponding author; e-mail: bibicu@infim.ro 
phonons to plasmons and magnons, and because of the incredible range of momentum and energy scales of interest, different probes must be used. Neutrons and $\mathrm{X}$-rays have been the weak-scattering probes of choice for the study of condensed matter systems at atomic scale.

Generally, it is simple to observe the elastic scattering with almost any source. The elastic intensities are high and confined to certain angles. Inelastic scattering is weak, diffuse in angle and difficult to resolve.

The total Rayleigh scattering intensity has an angular distribution dependent on the spatial atomic structure. A division between elastic and inelastic components depends on the thermal motion and on the correlations in the motions of different atoms. The inelastic scattering induces a line broadening of the scattered radiation. In order to explore extremely fine energy changes the incident radiation should have a very narrow line width. The detection of the scattered radiation via the nuclear resonance absorption, with an energy resolution of the order of $10^{-8} \mathrm{eV}$ for the $14.4 \mathrm{keV}$ transition of ${ }^{57} \mathrm{Fe}$, makes this technique very sensitive to dynamics changes. Therefore, Rayleigh scattering of the Mössbauer radiation (RSMR) is able to separate elastically and inelastically scattered radiation from systems not containing Mössbauer isotope [1-4]. In ordinary X-ray diffraction experiments, using X-ray tubes, such separation is impossible. The corrections are performed in these cases using theoretical models based on different approximations. The analytical methods calculate the thermal diffusion scattering (TDS) contribution neglecting the anisotropy of the elasticity and approximate the illuminated volume in reciprocal space either by a sphere or a cylinder. Computer programs that take into account an anisotropy of the elasticity and perform the integration in reciprocal space numerically are very time-consuming.

The relative complexity of the equipment required to perform RSMR measurements, as opposed to the common transmission and backscattering forms of the Mössbauer spectroscopy, is a barrier to the routine use of this technique. RSMR experiments are difficult essentially due to low brilliance of the Mössbauer sources, which limits the number of measured reflections.

This work presents the results of our RSMR dynamics studies on pyrolytic graphite $(\mathrm{C})$, a hexagonal crystalline structure with high mosaic divergence, and on rubidium tetrachlorozincate $\left(\mathrm{Rb}_{2} \mathrm{ZnCl}_{4}\right)$, an insulating orthorhombic crystal which forms an incommensurate superlattice below $303 \mathrm{~K}$. We measured the elastic and inelastic fractions of the Mössbauer radiation diffracted on pyrolytic graphite $\mathrm{C}(002)$ for different order of reflection at room temperature. The dependencies of the same intensities on the reflecting angle for the principal maximum of reflection are given. Careful measurements of recoil-less Rayleigh scattering of ${ }^{57} \mathrm{Fe}$ $14.4 \mathrm{keV}$ radiation on $\mathrm{Rb}_{2} \mathrm{ZnCl}_{4}$ crystals, at different temperatures around the normal-incommensurate phase transition point, provided valuable information on this transformation. We found a discontinuity in resonance effect $(\varepsilon)$ that proves a stepwise variation of the inelastic component of the scattered radiation. 


\section{Experimental}

The experimental setup for RSMR measurements built by us consists of three main parts: a goniometer, a Mössbauer spectrometer, and a personal computer. In our measurements we used a ${ }^{57} \mathrm{Co}$ Mössbauer source diffused into rhodium matrix. The detector was a proportional counter suitable for low gamma energies. The absorber used to analyze the Rayleigh scattered radiation was an iron enriched ${ }^{57} \mathrm{Fe}(30$ atomic \%) in a rhodium matrix (12 $\mu \mathrm{m}$ thickness and $15 \mathrm{~mm}$ diameter) placed between scatterer and detector. The sample and the source can be moved independently and in $(\theta, 2 \theta)$ manner by a stepping motor. A personal computer controls the orientation of the probe, the Doppler movement and performs the acquisition of the amplitude spectra and the Mössbauer spectra by means of three cards. Details of the experimental setup and the method used to discriminate the photons scattered elastically from those which undergoes inelastic scattering are described in [5].

The pyrolytic graphite has a hexagonal structure [6] with a high crystallites orientation. The mosaic divergence is approximately $1^{\circ}$. The crystal was mounted on a goniometer in such a way that the hexagonal plane $\mathrm{C}(002)$ was the scattering plane. All the measurements on the $\mathrm{C}(002)$ were performed at room temperature in symmetric Bragg geometry. The diffractometer was driven in $(\theta, 2 \theta)$ manner.

Rubidium tetrachlorozincate, $\mathrm{Rb}_{2} \mathrm{ZnCl}_{4}$, is one of the $\beta-\mathrm{K}_{2} \mathrm{SeO}_{4}$ type ferroelectrics which undergoes successive phase transitions: a second-order phase transition from normal orthorhombic phase $(\mathrm{N})$ to an incommensurate phase (I) at $T_{\mathrm{i}}=303 \mathrm{~K}$, and a first-order phase transition from the incommensurate phase to a ferroelectric commensurate $(\mathrm{C})$ phase at $T_{\mathrm{c}}=195 \mathrm{~K}$ [7]. The single crystals of $\mathrm{Rb}_{2} \mathrm{ZnCl}_{4}$ having the main face parallel with the $(a c)$ plane which designates the cleavage plane were fixed on a metallic holder, with $a$ direction as vertical axis. The heating of the sample is due by means of a resistive element. The temperature on sample surface was monitored during the measurements with a Chromel-Alumel thermocouple. The $14.4 \mathrm{keV}{ }^{57} \mathrm{Fe}$ photons from a Mössbauer source of ${ }^{57} \mathrm{Co}$ in rhodium, scattered at $5.82^{\circ}$ with respect to the (ac) plane, were measured at different temperatures around $T_{\mathrm{i}}$. In order to obtain a satisfactory statistics in a reasonable measuring time, the horizontal divergence of the beam was $2.8^{\circ}$.

The energy resolution of the setup was $10^{-8} \mathrm{eV}$. It depends on the sourceabsorber combination. All radiation scattered with a change in energy less than this value is considered as elastic one.

The diffraction curves were fitted by Gaussian functions while the Mössbauer spectra were fitted by Lorentzian functions with a computer program based on Marquardt's algorithm [8]. 


\section{Results and discussion}

Figure 1a, b presents in relative scales the diffraction and the absorption curves of $14.4 \mathrm{keV}$ Mössbauer radiation scattered on $\mathrm{C}(002)$ for the first three orders of diffraction. While the scattered intensities were registered making an angular scan around the maxima, in the case of the absorption spectra the crystal was fixed at the Bragg angles: $\theta_{\mathrm{BRAGG}}^{1}=7.38^{\circ}, \theta_{\mathrm{BRAGG}}^{2}=14.88^{\circ}, \theta_{\mathrm{BRAGG}}^{3}=22.66^{\circ}$, respectively. It is worthy noting that all the diffraction curves provide the same
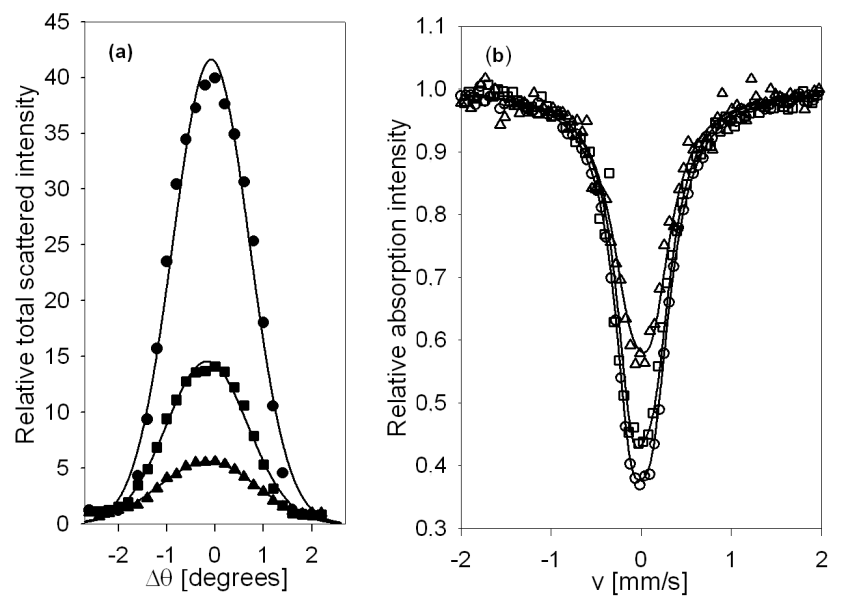

Fig. 1. The diffraction curves (a) (filled symbols) and absorption spectra (b) (open symbols) of the Mössbauer radiation scattered on $\mathrm{C}(002)$ for the first three orders $n$ of reflection. Circles: $n=1$, squares: $n=2$, triangles up: $n=3$.

line width but different intensities because as the angle scattering increases the proportion of coherent to non-coherent radiation diminishes [9]. The absorption spectra also present the same line widths but different resonance effects. The constancy of the line widths marks a structural stability. The resonance effects $\varepsilon$, proportional to the fraction of recoil-less $\gamma$-photons which are scattered elastically by the sample, were calculated with the relation

$$
\varepsilon=\frac{N_{\infty}-N_{0}}{N_{\infty}}
$$

where $N_{\infty}$ and $N_{0}$ represent the numbers of counts registered out of and in resonance conditions for the scattering geometry. More explicitly

$$
\begin{aligned}
& N_{\infty}=N_{\mathrm{el}}+N_{\text {in }}+N_{\mathrm{B}}, \\
& N_{0}=N_{\mathrm{el}}\left(1-\varepsilon_{0}\right)+N_{\mathrm{in}}+N_{\mathrm{B}},
\end{aligned}
$$

$N_{\text {el }}, N_{\text {in }}$ being the numbers of photons scattered elastically and respectively inelastically and $N_{\mathrm{B}}$ the background. The quantities 


$$
f_{\mathrm{el}}=\varepsilon / \varepsilon_{0} \quad \text { and } \quad f_{\text {in }}=1-\varepsilon / \varepsilon_{0}
$$

give the fractions of the incident radiation that are scattered elastically and inelastically, respectively, by the sample under investigation [2]. The effect $\varepsilon_{0}$ is registered without scatterer, in a transmission experiment. The decrease in the elastic fractions with the increase in the order of reflection is accompanied by an appropriate increase in the inelastic fractions.

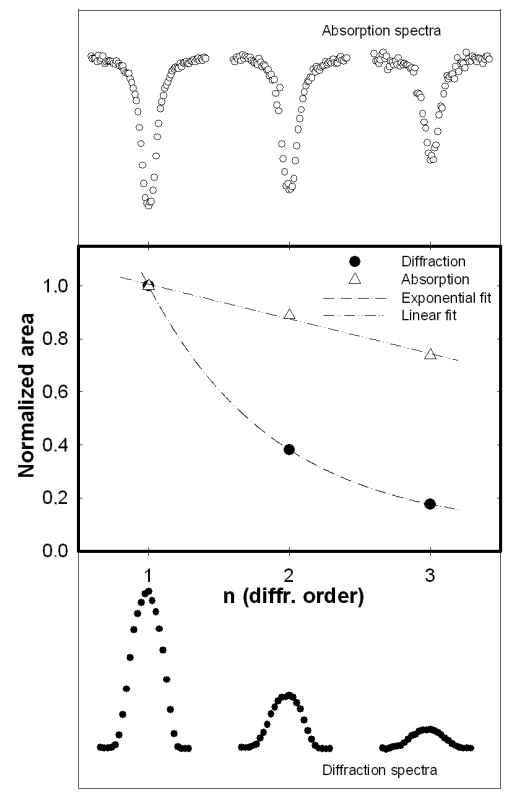

Fig. 2. Normalized area of the scattering and nuclear resonance absorption spectra versus different orders $n$ of diffraction for $\mathrm{C}(002)$.

The evolution with the order of diffraction (scattering angle) of the area under scattering and nuclear resonance absorption curves, respectively, is represented in normalized scales in Fig. 2. The exponential decay of the diffraction peaks areas is due to the Debye-Waller factor. The contribution of this factor consists in a reduction of the scattered intensity without a broadening of the lines when the scattering angle increases. While the areas of the diffraction peaks lie on an exponential decay, the areas of the absorption dips are fitted by a linear function. This linear behavior suggests that the drop is not so fast for the absorption spectra like for the diffraction one. It means that there is an incoherent scattered radiation, with no change in energy, which does not appear in the scattering curves but has an important contribution to the nuclear resonance absorption. Usually the Rayleigh scattering is coherent but it could include an incoherent component [10]. The presence of the incoherent scattering is due to a lack for the correlation between the atomic motions. 


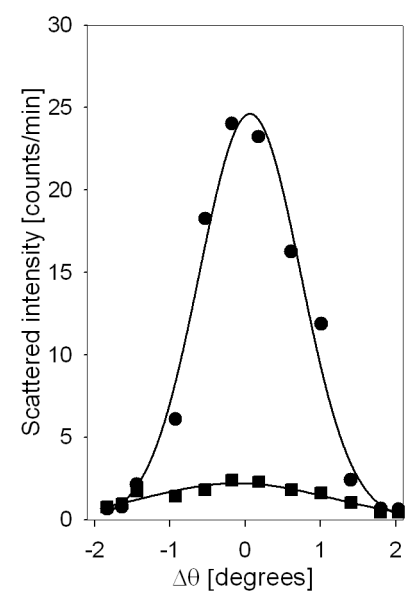

Fig. 3. Angular dependencies of elastic $(\bullet)$ and inelastic ( $\bullet$ intensities for $\mathrm{C}(002)$ principal maximum of diffraction. Symbols: experimental data, lines: Gaussian simulations. The error bars are included in the data symbols.

Figure 3 shows the angular dependencies of the elastic and inelastic intensities for the principal maximum of diffraction. The coherent inelastic intensity has a clear contribution to the total scattered intensity [5]. The smooth peak above the background, just under the Bragg elastic peak is a consequence of atomic motions coupling in the hexagonal plane of the carbon. In the case of one-phonon scattering processes, the differential cross-section for coherent inelastic scattering of photon is [11]:

$$
\frac{\mathrm{d} \sigma}{\mathrm{d} \Omega} \sim F^{2} \sum_{\boldsymbol{q} j} \frac{\left|\boldsymbol{Q} \cdot \boldsymbol{e}_{\boldsymbol{q} j}\right|^{2}}{\omega_{\boldsymbol{q} j}}
$$

where $F$ is the structure factor, $\boldsymbol{e}_{\boldsymbol{q} j}$ is the polarization vector of modulus unity for the lattice mode $(\boldsymbol{q}, j)$ characterized by the frequency $\omega_{\boldsymbol{q} j}$. The inelastic scattering intensity, proportional to $\left|\boldsymbol{Q} \cdot \boldsymbol{e}_{\boldsymbol{q} j}\right|^{2}$, has a maximum for modes polarized parallel to the scattering vector $\boldsymbol{Q}$ and zero for perpendicular polarization. Therefore the modes polarized perpendicular to the hexagonal plane of the carbon crystal have the main contribution to the coherent inelastic scattering.

The physical properties of $\mathrm{Rb}_{2} \mathrm{ZnCl}_{4}$ were extensively studied by different experimental techniques: X-ray diffraction, neutron scattering, nuclear magnetic resonance, Raman scattering, as well as some optical techniques [12]. Incommensurate phases occur in crystals when some local property develops a spatially periodic modulation with a wavelength, which is incommensurate with the underlying lattice spacing. In the high-temperature phase the crystal has a three-dimensional structure as an ordinary crystal. In the incommensurate phase, the symmetry group of the system is a superspace group. The translational periodicity, lost in the incommensurate phase, can be refund in the commensurate phase. 
The nuclear resonance absorption spectra of the radiation scattered on the (ac) plane with the crystal $a$ direction perpendicular on the rotation plane were measured at nine different temperatures $T$, between $289.5 \mathrm{~K}$ and $318 \mathrm{~K}$. The spectra were well fitted with a single Lorentzian line shape. Table gives the spectral parameters of the nuclear resonance absorption curves of the Mössbauer radiation scattered on $\mathrm{Rb}_{2} \mathrm{ZnCl}_{4}$. All spectra provide the same value for the line widths within experimental errors and practically equal to that obtained in a conventional transmission Mössbauer measurement. The absence of the nuclear resonance lines broadening means a structural stability on a time scale of the order of $10^{-7} \mathrm{~s}$, comparable with the lifetime of the excited level of the Mössbauer isotope $\left({ }^{57} \mathrm{Fe}\right)$. Time-resolved neutron studies revealed that the structural changes associated with the incommensurate-commensurate phase transition proceed on a millisecond time scale [13].

TABLE

Spectral parameters of the nuclear resonance absorption spectra of the Mössbauer radiation scattered on $\mathrm{Rb}_{2} \mathrm{ZnCl}_{4}$ and the ratio between inelastic and elastic fractions $\left(f_{\mathrm{in}} / f_{\mathrm{el}}\right)$.

\begin{tabular}{c|c|c|c|c|c}
\hline \hline Spectrum & $T[\mathrm{~K}]$ & $v_{0}[\mathrm{~mm} / \mathrm{s}]$ & $\Gamma[\mathrm{mm} / \mathrm{s}]$ & $\varepsilon[\%]$ & $f_{\text {in }} / f_{\mathrm{el}}[\%]$ \\
\hline S1 & 289.5 & $-0.034 \pm 0.004$ & $0.64 \pm 0.04$ & $22.8 \pm 0.4$ & $27.2 \pm 0.6$ \\
S2 & 294 & $-0.036 \pm 0.004$ & $0.65 \pm 0.04$ & $23.0 \pm 0.4$ & $26.3 \pm 0.6$ \\
S3 & 298 & $-0.033 \pm 0.005$ & $0.67 \pm 0.02$ & $23.4 \pm 0.4$ & $24.1 \pm 0.6$ \\
S4 & 301 & $-0.030 \pm 0.004$ & $0.67 \pm 0.01$ & $23.3 \pm 0.2$ & $24.5 \pm 0.6$ \\
S5 & 303 & $-0.036 \pm 0.008$ & $0.64 \pm 0.03$ & $21.3 \pm 0.5$ & $36.4 \pm 0.7$ \\
S6 & 305 & $-0.034 \pm 0.007$ & $0.66 \pm 0.02$ & $22.1 \pm 0.4$ & $31.4 \pm 0.7$ \\
S7 & 308 & $-0.025 \pm 0.007$ & $0.69 \pm 0.03$ & $21.6 \pm 0.4$ & $34.4 \pm 0.7$ \\
S8 & 313 & $-0.034 \pm 0.006$ & $0.65 \pm 0.02$ & $21.4 \pm 0.4$ & $35.5 \pm 0.7$ \\
S9 & 318 & $-0.030 \pm 0.004$ & $0.64 \pm 0.03$ & $21.6 \pm 0.2$ & $34.4 \pm 0.7$
\end{tabular}

The experimental values of $\varepsilon$ for different temperatures are presented in Fig. 4. A clear discontinuity is observed at $T_{\mathrm{i}}$.

Figure 5 shows the variation of the resonance area as a function of temperature. The normalization is made for the area registered at $318 \mathrm{~K}$, in the normal phase, far from the transition point. Approaching $T_{\mathrm{i}}$, the area decreases from both the low and high temperature sides and becomes minimum at $T_{\mathrm{i}}$. This variation unequivocally shows an anomaly in the vicinity of the $\mathrm{N}-\mathrm{I}$ transition temperature and suggests a drastic change in the scattered radiation. Analysis of the scattered photons by means of the resonance filter [14, 15] yields information about the fluctuation dynamics near the phase transition temperature. The behavior of the inelastic intensity in the critical region can be explained by qualitative considerations [16]. At large frequencies of fluctuations, far from the phase 


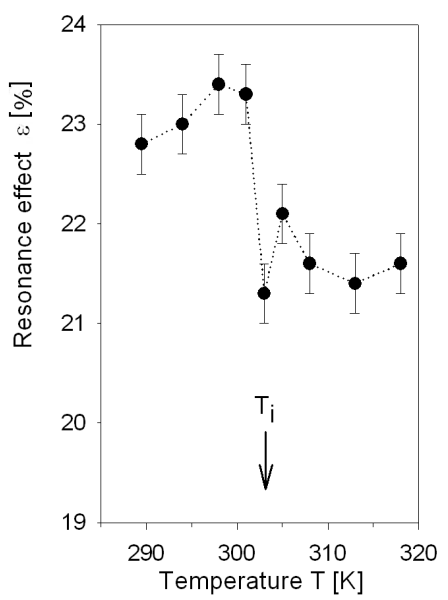

Fig. 4. Temperature dependence of the nuclear resonance effects $\varepsilon$ in $\mathrm{Rb}_{2} \mathrm{ZnCl}_{4}$; dotted curve is a guide line.

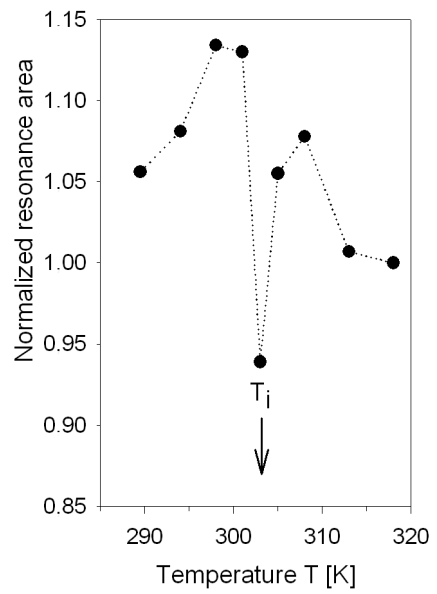

Fig. 5. Temperature variation of the normalized resonance area of $\mathrm{Rb}_{2} \mathrm{ZnCl}_{4}$; dotted curve is a guide line.

transition, a wide line, with width $\sim \omega_{\boldsymbol{q}}$, formed in the condensed medium during the Mössbauer radiation scattering, is studied with respect to the energy by a narrow resonance filter with the width $\Gamma$. Naturally, the ratio between the number of photons absorbed in resonant and non-resonant way, respectively, tends to be small and its temperature dependence is defined by the dependence of $\omega_{\boldsymbol{q}}(\tau)$, where $\tau=\left(T-T_{\mathrm{i}}\right) / T_{\mathrm{i}}$. In another extreme case, $\omega_{\boldsymbol{q}}$ much more smaller than $\Gamma$, the incident Mössbauer radiation beam is slightly modulated by the medium and so it does not practically contain the information on the behavior of the critical fluctuations. The most interesting case is $\omega_{q} \sim \Gamma$. In this region, the fluctuations 
being rather developed still have a sufficiently strong influence on the nature of the scattering. At the same time the energy width of the scattered beam is not too large in comparison with $\Gamma$.

Using the experimental values of $\varepsilon, N_{\infty}$, and $N_{\mathrm{B}}$, we calculated the elastic and inelastic fractions of the scattered radiation, $f_{\mathrm{el}}$ and $f_{\mathrm{in}}$, respectively. The temperature dependence of the ratio $f_{\text {in }} / f_{\text {el }}$ is shown in Fig. 6 . The ratio $f_{\text {in }} / f_{\text {el }}$ gives especially information on the dynamics of the lattice.

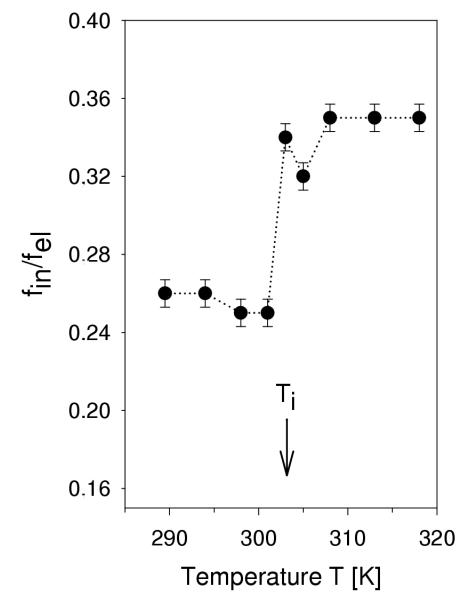

Fig. 6. Temperature dependence of the ratio $f_{\text {in }} / f_{\text {el }}$ of the Mössbauer radiation scattered on the $(a c)$ plane of $\mathrm{Rb}_{2} \mathrm{ZnCl}_{4}$; dotted curve is a guide line.

Two models have been proposed in order to describe the normal-incommensurate phase transition in insulators of the $\mathrm{A}_{2} \mathrm{BX}_{4}$ family: the displacive one and the order-disorder one [17]. The appearance in incommensurate phase of a modulation of the atoms positions with a periodicity which is an irrational fraction of the periodicity of the underlying lattice characterizes the displacive model. A critical soft mode, associated with the new order parameter related to this modulation, condenses out as the temperature decreases to $T_{\mathrm{i}}[18]$. In the order-disorder model the potential energy surface of the atoms is considered to have two minima in the incommensurate phase and the probability of finding an atom in a specific minimum is modulated. A critical mode is also associated to this transition mechanism and corresponds to large jumps of the atoms between the two positions. The critical scattering of the Mössbauer radiation could be due either to the photon-soft phonon interaction, in the displacive model, or to the photon-spin-wave interaction, in the case of the order-disorder transition model.

A soft mode was detected in the incommensurate phase of $\mathrm{Rb}_{2} \mathrm{ZnCl}_{4}$ by the Raman scattering technique [19] and elastic anomalies around $T_{\mathrm{i}}$ have been discussed taking it into account [20]. 
The critical scattering in the normal phase of $\mathrm{Rb}_{2} \mathrm{ZnCl}_{4}$ was studied by $\mathrm{X}$-ray scattering and the results were found to be consistent with the existence of the soft mode at $T>T_{\mathrm{i}}$ [21]. NMR measurements [22] provide another support for the existence of a soft mode in the normal phase of $\mathrm{Rb}_{2} \mathrm{ZnCl}_{4}$.

According to relation (3.4), the modes with low frequency have an important contribution to the $f_{\text {in }}$. The magnitude of the coherent inelastic intensity increases in general with decreasing $\boldsymbol{q}$.

Our results are consistent with the displacive model [23], which predicts a critical regime of the inelastic scattering of photons around $T_{\mathrm{i}}[21]$. The critical regime of the inelastic scattering is related to the critical soft mode whose contribution to the differential cross-section is important due to its frequency tending to zero. Due to the fact that in our experiment the scattering vector $\boldsymbol{Q}$ was normal to the $(a c)$ plane, the soft mode would oscillate perpendicular to this plane and would involve the motion of the $\mathrm{Rb}$ atoms, which are by far the most efficient scattering atoms of $\mathrm{Rb}_{2} \mathrm{ZnCl}_{4}$.

\section{Conclusions}

In the present paper we report on the results obtained by the Rayleigh scattering of the Mössbauer radiation dynamics studies in $\mathrm{C}$ and $\mathrm{Rb}_{2} \mathrm{ZnCl}_{4}$.

We found out that the scattering conditions (geometry, temperature) influence the resonance effect $\varepsilon$, which is a measure of the fraction of the recoil-less photons scattered elastically by the sample. The absence of the nuclear resonance lines broadening means the structural stability in a time scale of the order of $10^{-7} \mathrm{~s}$. The contribution of the coherent inelastic scattering to the total intensity measured in $\mathrm{C}(002)$ is interpreted as a consequence of the coupling motions of $\mathrm{C}$ atoms in the hexagonal plane. Moreover, we prove the presence of the incoherent scattered radiation, with no change in energy, due to a lack for the correlation between the atomic motions. Our experiments revealed a stepwise variation of the resonance effect at the normal-incommensurate transition temperature in $\mathrm{Rb}_{2} \mathrm{ZnCl}_{4}$ associated with a strong anomaly in the resonance area. This means that a significant change in the inelastic fraction of the radiation scattered on (ac) plane, and implicitly in the lattice dynamics, occurs at the normal-incommensurate phase transition in $\mathrm{Rb}_{2} \mathrm{ZnCl}_{4}$. The phase transformation is discussed in connection with the photonphonon interaction. Therefore the critical regime of the inelastic scattering of the Mössbauer radiation by the $(a c)$ plane is related to the soft mode, whose contribution to the differential cross-section is important due to its frequency approaching zero at the transition point.

The obtained results demonstrate that the RSMR technique provides the high-energy resolution able to evidence extremely small changes in the energy of the scattered radiation due to the interaction with the sample. With an energy resolution of $10^{-8} \mathrm{eV}$, RSMR is a powerful tool for dynamics investigations in samples not containing the Mössbauer isotopes. 


\section{Acknowledgments}

This work was partially supported for National Institute for Material Physics by governmental project "New Directions in Condensed Matter Physics and Material Physics" (COMAFI).

\section{References}

[1] G. Albanese, C. Ghezzi, A. Merlini, Phys. Rev. B 1, 65 (1973).

[2] G. Albanese, A. Deriu, Riv. del Nuovo Cimento 2, 1 (1979).

[3] K. Krec, W. Steiner, M. Wada, Acta Crystallogr. A 49, 198 (1993).

[4] G. Albanese, A. Deriu, F. Cavatorta, A. Rupprecht, Hyperfine Interact. 95, 97 (1995).

[5] S.E. Enescu, I. Bibicu, V. Zoran, A. Kluger, A.D. Stoica, V. Tripadus, Eur. Phys. J. Appl. Phys. 3, 119 (1998).

[6] R. Al-Jishi, G. Dresselhaus, Phys. Rev. B 26, 4514 (1982).

[7] P. Mischo, F. Decker, U. Häcker, K.P. Holzer, J. Petersson, Phys. Rev. Lett. 78, 2152 (1997).

[8] Sanda E. Enescu, Rom. Rep. Phys. 47, 585 (1995).

[9] L. Bragg, The Crystalline State, Vol. 1, G. Bell and Sons, London 1955, p. 208.

[10] D.C. Champeney, Rep. Prog. Phys. 42, 1017 (1979).

[11] B. Donovan, J.F. Angress, Lattice Vibrations, Chapman and Hall, London 1971, p. 127.

[12] H.Z. Cummins, Phys. Rep. 185, 212 (1990).

[13] G. Eckold, M. Hagen, U. Steigenberger, Physica B 234-236, 151 (1997).

[14] C.N.W. Darlington, W.J. Fitzgerald, D.A. O'Connor, Phys. Lett. A 54, 35 (1975).

[15] C.N.W. Darlington, D.A. O'Connor, J. Phys. C, Solid State Phys. 9, 3561 (1976).

[16] E.V. Zolotojabko, V.N. Kashcheev, J. Phys. C, Solid State Phys. 10, 4599 (1977).

[17] C.J. de Pater, C. van Dijk, Phys. Rev. B 18, 1281 (1978).

[18] S. Zumer, R. Blinc, J. Phys. C, Solid State Phys. 14, 465 (1981).

[19] M. Wada, A. Sawada, Y. Ishibashi, J. Phys. Soc. Japan 45, 1429 (1978).

[20] S. Hirotsu, K. Toyota, K. Hamano, J. Phys. Soc. Japan 46, 1389 (1979).

[21] S.R. Andrews, H. Mashiyama, J. Phys. C, Solid State Phys. 16, 4985 (1983).

[22] R. Blinc, B. Lozar, V. Rutar, S. Zumer, Solid State Commun. 42, 679 (1982).

[23] S.E. Enescu, I. Bibicu, M.N. Grecu, A. Kluger, Solid State Commun. 111, 299 (1999). 